\title{
The application of a hybrid model for identifying and ranking indicators for assessing the sustainability of wastewater treatment systems
}

\begin{abstract}
This paper proposes a combination of the Fuzzy Delphi and Fuzzy Analytic Hierarchy Process (FAHP) as an efficient tool for dealing with complex decision-making problems and to prove that integrating the Fuzzy Delphi and FAHP modeling is an objective and practical process. The application of these methods allows a large amount of information to be aggregated in a rigorous manner. The suggested fuzzy set theory helps to represent the uncertainty and vagueness of human's subjective thinking process in dealing with decision problems. This hybrid approach enables decision-makers to evaluate priorities more efficiently and objectively and make the decision process more reliable. Therefore, the main goal of the present research is to develop this decision support approach to identify the key evaluation criteria and indicators in the process of selecting industrial wastewater treatment technology (WTT) from a sustainability perspective based on expert opinion and questionnaires. Since a realistic evaluation needs to be conducted in an actual condition, Iran's steel industry was selected as the case study. In this research, based on the findings of the literature review and Fuzzy Delphi method screening, several evaluation criteria and indicators are identified. Then, the FAHP is employed to examine their relationships under a hierarchy structure and to determine their weights and priorities. The findings of this paper can be applied as a reference for the steel industry in decision-making for the selection of the optimal wastewater treatment technology. In addition, other industries can apply this feasible and practical approach in their decision-making process with respect to their differences in capacities, limitations, wastewater characteristics and local conditions.
\end{abstract}

Keyword: Sustainability assessment; Criteria and indicators; Wastewater treatment technology; Fuzzy Delphi; FAHP 